\title{
Actinohivin: specific amino acid residues essential for anti-HIV activity
}

\begin{abstract}
Atsushi Takahashi ${ }^{1}$, Junji Inokoshi ${ }^{2}$, Masaru Tsunoda ${ }^{1}$, Kaoru Suzuki ${ }^{1}$, Akio Takenaka ${ }^{1}$, Takeshi Sekiguchi ${ }^{1}$, Satoshi Omura ${ }^{3}$ and Haruo Tanaka ${ }^{1}$

Actinohivin (AH) is a microbial lectin containing 114 amino acids, which inhibits human immunodeficiency virus (HIV) infection. This effect is brought about by its specific binding to Man- $\alpha$ (1-2)-Man unit(s) of high-mannose type glycan (HMTG) bound to HIV gp120. The recently determined crystal structure of AH suggests that three repeated segments (the residue numbers 1-38, 39-76 and 77-114 for segments 1,2 and 3, respectively) form three sugar-binding pockets to accommodate Man- $\alpha$ (1-2)-Man units. The strong specific binding of AH to gp120 is considered to be due to multivalent interaction of the three sugar-binding pockets with three HMTGs of gp120 via the 'cluster effect' of lectin. It remains to be seen which residues of the sugar-binding pockets are essential for acceptance of Man- $\alpha(1-2)-$ Man. To identify the amino acid residues critical for anti-HIV effect, we performed mutational analysis. Mutant AHs were subjected to enzyme-linked immunosorbent assay testing for gp120-binding activity and to syncytium formation assay. As a result, it was revealed that Asp15, Tyr23, Leu25, Asn28 and Tyr32 in segment 1, Tyr61 in segment 2 and Tyr99 in segment 3 are essential for anti-HIV activity. The conserved residues, Asp53, Leu63, Asn66 and Tyr70, in segment 2 and, Asp91, Leu101, Asn104 and Tyr108, in segment 3 are also necessary. Furthermore, aromatic residues at positions 23 and 32 are required for creation of potency. These data will be useful for predicting the detailed mechanism of AH-Man- $\alpha(1-2)-M a n / H M T G / g p 120$ interaction by computational analysis and for possible development of more potent microbicides for prevention of HIV transmission.
\end{abstract}

The Journal of Antibiotics (2010) 63, 661-665; doi:10.1038/ja.2010.106; published online 15 September 2010

Keywords: actinohivin; anti-HIV lectin; cluster effect of lectin; gp120-binding site; HMTG-binding site; mannose-binding site; microbicide

\section{INTRODUCTION}

In 2008, the number of people living with human immunodeficiency virus (HIV) worldwide exceeded 31 million, and 2.7 million people were newly infected with HIV, mainly via heterosexual intercourse. ${ }^{1}$ AIDS-related deaths rose to 2 million in 2008. In recent years, although the number of new HIV infection has tended to decline, ${ }^{1}$ it is important to continue efforts to prevent the global spread of HIV infection. Although a vaccine would provide the most effective prophylaxis, the high mutational rate of HIV is a major factor in making development of a vaccine difficult. Other means of prophylaxis, such as microbicides, ${ }^{2}$ are urgently required.

HIV entry into susceptible cells is initiated by binding of the viral envelope glycoprotein gp120 to cellular receptors, CD4/CXCR4 or CCR5. The HIV gp120 is highly glycosylated, about half of its molecular weight being accounted for by carbohydrate moieties. Recombinant gp120 from HIV-1 $1_{\text {IIIB }}$ expressed in Chinese hamster ovary cells reveals that the carbohydrate moieties are composed of 13 complex-type glycans and 11 hybrid or high-mannose type glycans
(HMTGs). ${ }^{3}$ These glycans contribute to the low antigenicity of HIV gp120 by hiding epitopes responsible for HIV infectivity. ${ }^{4}$ In addition to a high mutational rate of HIV, the weak neutralizing antibody response of HIV gp120 also makes vaccine development problematic. $^{5}$

Recently, lectins targeting the glycans of HIV gp120 have become attractive candidates as anti-HIV agents. ${ }^{6,7}$ Lectins bound to glycans inhibit the HIV entry process, causing steric hindrance between gp120-receptor interaction and by preventing the conformational changes of gp120. They can inhibit not only the passage of virus into target cells but also syncytium formation between virus-infected and -uninfected cells. This bifunctional mode of lectins is considered to be a useful property of potential use in topical microbicides.

Actinohivin (AH) is a potent anti-HIV lectin produced by an actinomycete, Longispora albida gen. nov., sp. nov. ${ }^{8}$ It inhibits the entry of various HIV-1 and HIV-2 strains into susceptible cells $\left(\mathrm{IC}_{50}=2-110 \mathrm{nM}\right)$, as well as T-tropic and M-tropic syncytium formation $\left(\mathrm{IC}_{50}=60\right.$ and $\left.700 \mathrm{~nm}\right) .{ }^{9}{ }^{9} 10$ This inhibitory effect of $\mathrm{AH}$ is brought

\footnotetext{
${ }^{1}$ Faculty of Pharmacy and College of Science and Engineering, Iwaki Meisei University, Fukushima, Japan; ${ }^{2}$ School of Pharmacy, Kitasato University, Tokyo, Japan and ${ }^{3}$ Kitasato Institute for Life Sciences, Kitasato University, Tokyo, Japan

Correspondence: Professor H Tanaka, Faculty of Pharmacy and College of Science and Engineering, Iwaki Meisei University, 5-5-1 Chuodai-lino, Iwaki, Fukushima 970-8551, Japan. 
about by potent binding to HIV gp120 through specific interaction with Man- $\alpha(1-2)$-Man units of HMTGs on gp120. ${ }^{11,12}$

$\mathrm{AH}$ is composed of 114 amino acid residues and contains three tandem repeats (amino acids 1-38, 39-76 and 77-114; segments 1, 2 and 3, respectively), with each segment containing well-conserved LD-QXW motifs. These profiles suggest that AH belongs to the carbohydrate-binding module family 13 (CBM13). The three-dimensional structure of proteins classified into CBM13 shows a pseudothreefold symmetry ( $\beta$-trefoil fold $\left.{ }^{13}\right)$, in accordance with the observed three tandem repeats. Each repeat involves a sugar-binding pocket formed by an LD-QXW sequence. The sugar-binding specificities of the proteins are different from each other and LD-QXW sequences of AH form Man- $\alpha(1-2)$-Man-binding pockets, whereas those of ricin toxin B-chain (RTB) from Ricinus communis ${ }^{14}$ or xylan-binding domain (XBD) of $\beta$-xylanase from Streptomyces olivaceoviridis $\mathrm{E}-86,{ }^{15,16}$ also belonging to CBM13, form galactose or xylose oligomer-binding pockets, respectively.

The galactose or xylose oligomer-binding residues of RTB or XBD have been identified and are involved in LD-QXW sequences, while it remains unknown which residues of AH participate in Man- $\alpha(1-2)-$ Man binding. Sugar-binding pockets of AH are formed by LD-QXW sequences and Man- $\alpha(1-2)$-Man-binding residues essential for anti-HIV activity are believed to exist in these regions.

This paper identifies some residues important for activity, through mutational analysis of amino acid residues based on a multiple sequence alignment between AH, RTB and XBD. Our results define the amino acid residues essential for the specific interaction between AH and Man- $\alpha$ (1-2)-Man/HMTG/gp120, consistent with X-ray structures, ${ }^{12}$ and should facilitate development of highly active $\mathrm{AH}$ derivatives for use in anti-HIV microbicides.

\section{MATERIALS AND METHODS \\ PCR}

PCR was carried out using a $0.2 \mathrm{~mm}$ deoxynucleoside triphosphate mixture, Takara LA Taq buffer (Takara Bio, Tokyo, Japan), $0.25 \mathrm{~mm} \mathrm{MgCl}_{2}, 5 \%$ DMSO, $50 \mathrm{pmol}$ of each primer and $0.5 \mathrm{U}$ Takara LA Taq DNA polymerase (Takara Bio), and $10 \mathrm{ng}$ of pBluescript II SK+: $2 \mathrm{~A} 3 \mathrm{~K}(\mathrm{pBSK}+: 2 \mathrm{~A} 3 \mathrm{~K})^{17}$ or PCR products as a template. The reaction was run for 30 cycles, incorporating denaturing at $94^{\circ} \mathrm{C}$ for $30 \mathrm{~s}$, annealing at $60^{\circ} \mathrm{C}$ for $30 \mathrm{~s}$ and polymerization at $72^{\circ} \mathrm{C}$ for $1 \mathrm{~min}$ for each cycle. The amplified DNA fragments were purified by agarose gel electrophoresis.

\section{Construction of mutant $\mathrm{AH}$ expression vectors}

Mutant AH genes were generated by PCR through the technique of splicing by overlap extension. ${ }^{18}$ The sequences of primers used for the construction of each mutant $\mathrm{AH}$ gene and substitution positions of amino acid residue are shown in Table 1. First, PCR was run using AA-1 LIC (5'-GGTATTGAGGGTCGCGCCT CGGTGACCATC- $3^{\prime}$ ) and anti-sense primer, and sense primer plus TGA-114 LIC (5'-AGAGGAGAGTTAGAGCCTCAGCCGGTGTAC-3') as the primer sets. Second, PCR was carried out using AA-1 LIC and TGA-114 LIC as a primer set, and 1 pmol each of two DNA fragments obtained from the first PCR as templates, to yield full-length mutant $\mathrm{AH}$ genes. The genes purified by agarose gel electrophoresis were joined to a pET30 Xa/LIC vector (Merck, KGaA, Darmstadt, Germany), according to the manufacturer's protocol. The construction was amplified into Escherichia coli JM109 to confirm the nucleotide sequences and then transferred into E. coli BL21 (DE3) pLysS.

\section{Expression and purification of mutant AHs}

E. coli BL21 (DE3) pLysS, containing the mutant AH expression vector, was cultured in LB medium containing $30 \mu \mathrm{g} \mathrm{ml}^{-1}$ kanamycin, $34 \mu \mathrm{g} \mathrm{ml}^{-1}$ chloramphenicol and $2 \%$ glucose at $37^{\circ} \mathrm{C}$. The overnight culture was inoculated in a final concentration of $1 \%$ into fresh LB medium containing the above antibiotics and cultured at $37^{\circ} \mathrm{C}$. When the culture reached the appropriate
Table 1 Primer sets used for the construction of mutant AH genes

\begin{tabular}{|c|c|c|}
\hline $\begin{array}{l}\text { Mutant } \\
\text { (amino acid } \\
\text { substitution) }\end{array}$ & $\begin{array}{l}\text { Sense primer } \\
\text { sequence }\left(5^{\prime} \rightarrow 3^{\prime}\right)\end{array}$ & $\begin{array}{l}\text { Anti-sense primer } \\
\text { sequence }\left(5^{\prime} \rightarrow 3^{\prime}\right)\end{array}$ \\
\hline-1 (D15A) & CGCCTGCTGGCCAGCAACTAC & GTAGTTGCTGGCCAGCAGGCG \\
\hline$H(N 17 A)$ & CTGGACAGCGCCTACAACGGC & GCCGTTGTAGGCGCTGTCCAG \\
\hline$H(Y 18 A)$ & GACAGCAACGCCAACGGCAAC & GTTGCCGTTGGCGTTGCTGTC \\
\hline $\mathrm{AH}(\mathrm{N} 19 \mathrm{~A})$ & AGCAACTACGCCGGCAACGTC & GACGTTGCCGGCGTAGTTGCT \\
\hline$H(N 21 A)$ & CTACAACGGCGCCGTCTACACG & 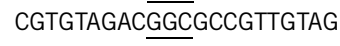 \\
\hline$H(Y 23 A)$ & GGCAACGTCGCCACGCTGCCC & GGGCAGCGTGGCGACGTTGCC \\
\hline $\mathrm{AH}(\mathrm{Y} 23 \mathrm{~F})$ & GGCAACGTCTTCACG CTGCCC & GGGCAGCGTGAAGACGTTGCC \\
\hline $\mathrm{AH}(\mathrm{Y} 23 \mathrm{~W})$ & GGCAACGTCTGGACGCTGCCC & GGGCAGCGTCCAGACGTTGCC \\
\hline$H(L 25 A)$ & GTCTACACGGCGCCCGCCAAC & GTTGGCGGGCGCCGTGTAGAC \\
\hline $\mathrm{AH}(\mathrm{N} 28 \mathrm{~A})$ & CTGCCCGCCGCCGGCGGGAAC & GTTCCCGCCGGCGGCGGGCAG \\
\hline $\mathrm{AH}(\mathrm{N} 31 \mathrm{~A})$ & CAACGGCGGGGCCTACCAGCGG & CCGCTGGTAGGCCCCGCCGTTG \\
\hline $\mathrm{AH}(\mathrm{Y} 32 \mathrm{~A})$ & GGCGGGAACGCCCAGCGGTGG & CCACCGCTGGGCGTTCCCGCC \\
\hline $\mathrm{AH}(\mathrm{Y} 32 \mathrm{~W})$ & GGCGGGAACTGGCAGCGGTGG & CCACCGCTGCCAGTTCCCGCC \\
\hline $\mathrm{AH}(\mathrm{Y} 61 \mathrm{~A})$ & GGCGCCGTCGCCACGCTGCCG & CGGCAGCGTGGCGACGGCGCC \\
\hline AH (Y99A) & GGCAACGTGGCCACACTGCCG & CGGCAGTGTGGCCACGTTGCC \\
\hline
\end{tabular}

Abbreviation: $\mathrm{AH}$, actinohivin.

Underlines show sequences of substituted amino acids residues.

density $\left(\mathrm{OD}_{600}=0.3\right)$, isopropyl-thio- $\beta$-D-galactopyranoside was added to a final concentration of $1 \mathrm{~mm}$ to induce the expression of His-tagged mutant $\mathrm{AH}$. The cells were cultured for a further $2 \mathrm{~h}$ and collected by centrifugation at $8000 \mathrm{~g}$ for $15 \mathrm{~min}$. The precipitated cells were washed with phosphate-buffered saline and suspended with binding buffer $(5 \mathrm{~mm}$ imidazole, $0.5 \mathrm{M} \mathrm{NaCl}$ and $125 \mathrm{~mm}$ Tris-HCl, $\mathrm{pH}$ 8.0). After disruption by sonic treatment in an ice bath, the cell lysate was centrifuged $(10000 \mathrm{~g}$ for $15 \mathrm{~min})$ to obtain the insoluble fraction containing mutant AHs. The insoluble fraction was dissolved in denaturing buffer (binding buffer containing $6 \mathrm{M}$ guanidine-HCl) and loaded onto a column $(10 \times 25 \mathrm{~mm})$ containing metal-chelating Sepharose $4 \mathrm{~B}$ (GE Healthcare, Uppsala, Sweden) previously activated by $\mathrm{Ni}^{2+}$ and equilibrated with denaturing buffer. The column was washed with a five-column volume of the same buffer and His-tagged mutant $\mathrm{AH}$ was eluted by denaturing buffer containing $60 \mathrm{~mm}$ imidazole. Finally, mutant AH was purified by HPLC using an ODS column (Senshu Pak Pegasil ODS, $6 \times 250 \mathrm{~mm}$, Senshu Scientific, Tokyo, Japan) under the same conditions, as previously described. ${ }^{17}$

\section{Protein determination}

Protein concentration was determined by the bicinchoninic acids (BCA) protein assay reagent (Pierce Biotechnology, Rockford, IL, USA). Bovine serum albumin was used as a standard.

\section{SDS-polyacrylamide gel electrophoresis}

All mutant AHs were analyzed by SDS- $12.5 \%$ polyacrylamide gel electrophoresis performed in a Tris-glycine buffer system. ${ }^{19}$ Each sample ( $5 \mu \mathrm{g}$ per lane) was mixed with SDS-polyacrylamide gel electrophoresis sample buffer $(125 \mathrm{mM}$ Tris-HCl (pH 6.8), 2.5\% SDS, 20\% glycerol, $0.01 \%$ bromophenol blue, $2.5 \mathrm{~mm}$ EDTA and $200 \mathrm{~mm}$ dithiothreitol), and heated at $95^{\circ} \mathrm{C}$ for $5 \mathrm{~min}$. The gel was stained using $0.05 \%$ Coomassie brilliant blue G-250.

\section{Examination of syncytium formation inhibitory activity}

Anti-syncytium formation activity was measured using HeLa/T-env/Tat and HeLa/CD4/Lac-Z cells as previously described. ${ }^{9} \mathrm{HeLa} / \mathrm{T}-\mathrm{env} / \mathrm{Tat}$ and HeLa/ $\mathrm{CD} 4 / \mathrm{Lac}-\mathrm{Z}$ cell cultures (each $8 \times 10^{3}$ cells per $50 \mu \mathrm{l}$ ) were co-cultivated with $10 \mu \mathrm{l}$ of a test compound solution in 96-well microtiter plates under $5 \% \mathrm{CO}_{2}$ at $37^{\circ} \mathrm{C}$ for $24 \mathrm{~h}$. $\beta$-Galactosidase activity in the cells was measured using $o$-nitrophenyl- $\beta$-D-galactopyranoside as a substrate. His-tagged mutant AHs were dissolved in $50 \%$ ethanol to make a concentration of $5 \mathrm{~mm}$ and then serially diluted with Dulbecco's modified Eagle's medium to the desired concentrations. 
Enzyme-linked immunosorbent assay for interaction between mutant AHs and gp120

Enzyme-linked immunosorbent assay was performed as previously described..$^{20}$ Briefly, $100 \mathrm{ng}$ per well of $\mathrm{HIV}_{\text {IIIB }}$ gp120 was bound to a 96-well protein adsorbing plate. Various concentrations of test samples were added to a plate and mutant AHs bound to gp120 were determined by incubation, first with anti-His antibody (GE Healthcare) and then incubation with anti-mouse Ig linked to horseradish peroxidase (GE Healthcare). Plates were then incubated with o-phenylenediamine dihydrochloride (OPD) solution $(16 \mathrm{~mm}$ citrate, $50 \mathrm{mM} \mathrm{Na}_{2} \mathrm{HPO}_{4}, 0.45 \mathrm{mg} \mathrm{ml}^{-1} \mathrm{OPD}$ and $0.0125 \% \mathrm{H}_{2} \mathrm{O}_{2}$ ) and bound mutant AHs were measured by determining absorbance at $492 \mathrm{~nm}$.

\section{RESULTS}

Multiple sequence alignment between AH, RTB and XBD

Figure 1 shows a multiple sequence alignment between AH, RTB and XBD. The Asp22, Gln35, Trp37 and Asn46 residues of RTB $1 \alpha$ domain, and the Asp325, Asn328, Gln338, Tyr340, His343 and Asn347 residues of XBD $\alpha$ domain, found in the LD-QXW sequences were identified as the galactose and the xylose oligomer-binding sites, respectively. ${ }^{14,16}$ The corresponding residues, Asp15, Tyr23, Leu25 and Tyr32, of AH the segment 1 are conserved in RTB and XBD. In addition, the Tyr 18 and Asn 28 residues of $\mathrm{AH}$ are also conserved in the xylose oligomer-binding sites of XBD. Mutational analysis of the amino acid residues was performed to investigate their contributions to Man- $\alpha(1-2)$-Man binding of AH. Although the polar residues, Asn17, Asn19, Asn21 and Asn31 of $\mathrm{AH}$, are not conserved in the sugar-binding sites of RTB and XBD, these were mutated to examine their effects.

Effect of amino acid substitution of AH LD-QXW sequence on gp120 binding and syncytium formation

All the recombinant mutants of $\mathrm{AH}$ are fusion proteins carrying additional 47 amino acid residues containing a His-tag at their $\mathrm{N}$-termini. ${ }^{17}$ The proteins were highly purified by metal-chelating Sepharose 4B and HPLC (as shown in Figure 2) as single bands on SDS-polyacrylamide gel electrophoresis. The syncytium formation assay (Table 2) shows that the substitutions of Ala for Asn17, Tyr18, Asn19, Asn21 and Asn31 have minimal affect on activity, compared with that of His-AH, suggesting that these residues are not involved in HMTG binding. However, the substitutions for Asp15, Tyr23, Leu25, Asn 28 and Tyr 32 cause drastic changes, lowering activity up to 20 -fold that of His-AH (Table 2). Syncytium formation assays also show the same trend in the gp120-binding activity, as seen in Figure 3a. Therefore, the latter five residues, Asp15, Tyr23, Leu25, Asn28 and Tyr32, appear to be essential for gp120 binding and inhibition of syncytium formation. As described in a previous paper, $G \ln 33$ is also essential. ${ }^{20}$ In total, six residues of segment 1 are involved in binding to HMTG. In order to confirm that the three segments are equivalent to each other in gp120 binding, Tyr61 of segment 2 and Tyr99 of segment 3 were mutated. Our data (shown in Table 2) indicate that these residues behave like the Tyr23 of segment 1 .

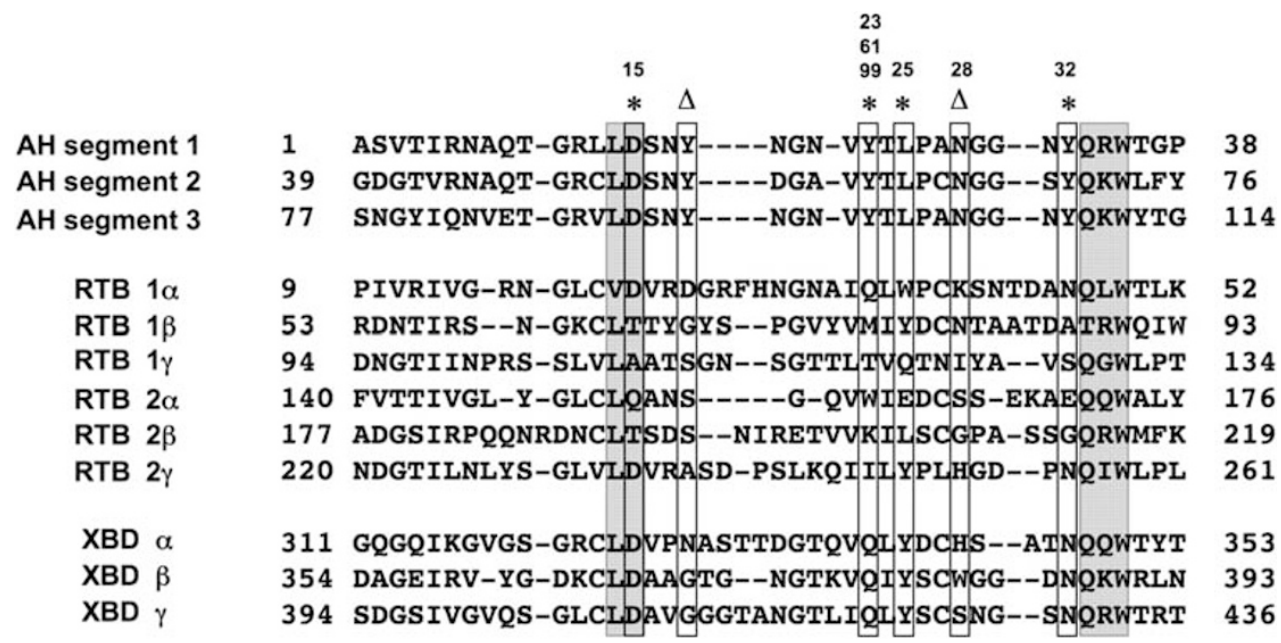

Figure $1 \mathrm{~A}$ multiple sequence alignment between actinohivin (AH), ricin toxin B-chain (RTB) and xylan-binding domain (XBD) (by ClustalW 1.83). Asterisks show the galactose-binding sites of RTB $1 \alpha$ domain and the xylose oligomer- binding sites of XBD $\alpha$ domain. Open triangles indicate xylose oligomer-binding sites of XBD $\alpha$ domain alone. The LD and QXW motifs are indicated in gray.

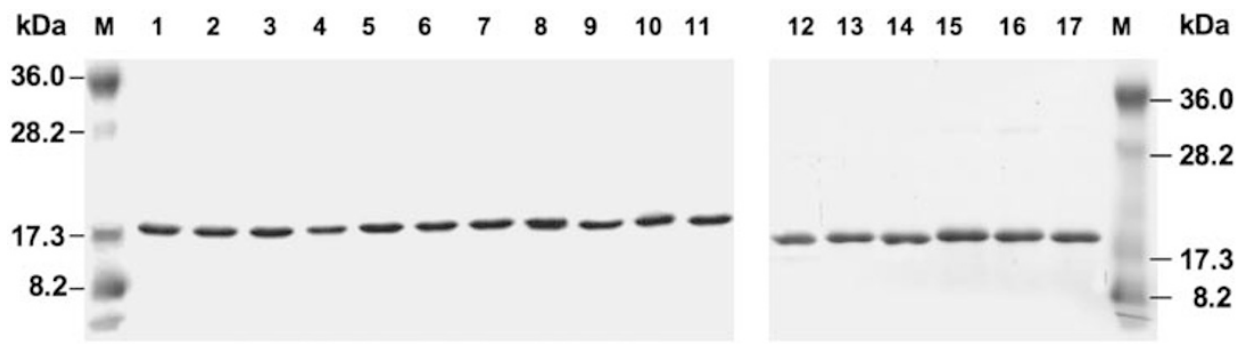

Figure 2 SDS-polyacrylamide gel electrophoresis of mutant actinohivins (AHs). Lane 1, His-AH; lane 2, His-AH (D15A); lane 3, His-AH (N17A); lane 4, HisAH (Y18A); lane 5, His-AH (N19A); lane 6, His-AH (N21A); lane 7, His-AH (Y23A); lane 8, His-AH (L25A); lane 9, His-AH (N28A); lane 10, His-AH (N31A); lane 11, His-AH (Y32A); lane 12, His-AH; lane 13, His-AH (Y23W); lane 14, His-AH (Y23F); lane 15, His-AH (Y32W); lane 16, His-AH (Y61A); lane 17, His-AH (Y99A); M, kaleidoscope polypeptide standards (Bio-Rad Laboratories, Hercules, CA, USA). 
Aromatic rings are required for potent activity at positions 23 and 32

The Trp37 residue of the RTB $1 \alpha$ domain was identified to be crucial for galactose binding. ${ }^{14}$ The X-ray structures show a galactose ring

Table 2 Anti-syncytium formation activity of mutant AHs

\begin{tabular}{lr}
\hline Mutant (amino acid substitution) & \multicolumn{1}{c}{$\mathcal{I}_{50}(\mu)^{\mathrm{a}}$} \\
\hline His-AH & $0.54 \pm 0.02$ \\
His-AH (D15A) & $7.21 \pm 0.45$ \\
His-AH (N17A) & $0.78 \pm 0.10$ \\
His-AH (Y18A) & $0.93 \pm 0.09$ \\
His-AH (N19A) & $0.61 \pm 0.13$ \\
His-AH (N21A) & $0.95 \pm 0.20$ \\
His-AH (Y23A) & $12.21 \pm 0.73$ \\
His-AH (L25A) & $2.32 \pm 0.20$ \\
His-AH (N28A) & $6.32 \pm 0.21$ \\
His-AH (N31A) & $0.74 \pm 0.24$ \\
His-AH (Y32A) & $7.29 \pm 0.57$ \\
His-AH (Y23W) & $3.91 \pm 0.31$ \\
His-AH (Y23F) & $3.74 \pm 0.12$ \\
His-AH (Y32W) & $0.75 \pm 0.02$ \\
His-AH (Y61A) & $6.23 \pm 0.54$ \\
His-AH (Y99A) & $10.12 \pm 0.88$
\end{tabular}

Abbreviation: $\mathrm{AH}$, actinohivin.

aAverage \pm s.d. of three repeated experiments.

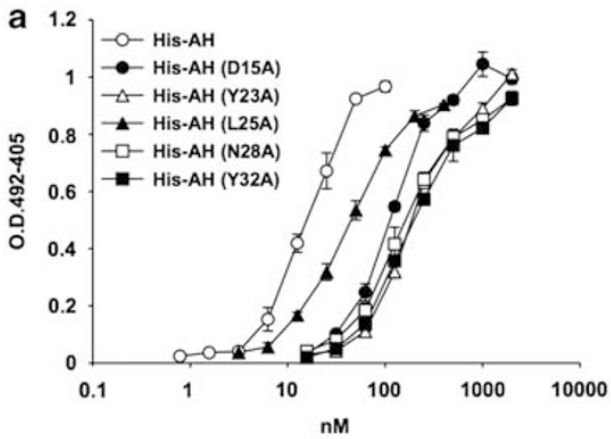

stacked on the aromatic ring of Trp37. Such stacking interaction between a pyranose and an aromatic ring is well established in carbohydrate-binding proteins. ${ }^{21,22}$ In the case of $\mathrm{AH}$, however, the two Tyr23 and Tyr32 residues might be required for gp 120 binding through stacking interactions with two mannose rings of Man- $\alpha(1-2)-M a n$. To confirm this speculation, aromatic amino acids, Trp and Phe, were substituted for the Tyr residues and their activities were assayed. The mutant His-AH (Y32W) shows no significant effect on anti-syncytium formation activity (Table 2) and on gp120-binding activity (Figure 3b). The activity of the mutant His- $\mathrm{AH}(\mathrm{Y} 23 \mathrm{~W})$ is reduced $\left(\mathrm{IC}_{50} \approx 3.8 \mu \mathrm{M}\right)$. Another mutant His-AH (Y23F) also shows low activity, similar to those of His-AH (Y23W).

Significantly, when compared with these mutants, the activity of His-AH (Y23A) is decreased at 3.2 -fold $\left(\mathrm{IC}_{50} \approx 12 \mu \mathrm{M}\right)$. Our results suggest that the side chains at positions 23 and 32 are required to have flat, bulky groups, such as aromatic groups. In addition, the hydroxyl group of Tyr at position 23 might be involved in the highly specific interaction with mannose moieties.

\section{DISCUSSION}

The microbial lectin AH contains 114 amino acids and consists of three highly conserved segments (the residue numbers 1-38, 39-76 and 77-114 for segment 1, 2 and 3, respectively). Each segment forms a structural module composed of four or five $\beta$-strands, a $\pi$-helix and a long loop, as shown in Figure $4 \mathrm{a}^{12}$ A valley (10 $\mathrm{A}$ in length, $15 \AA$ in

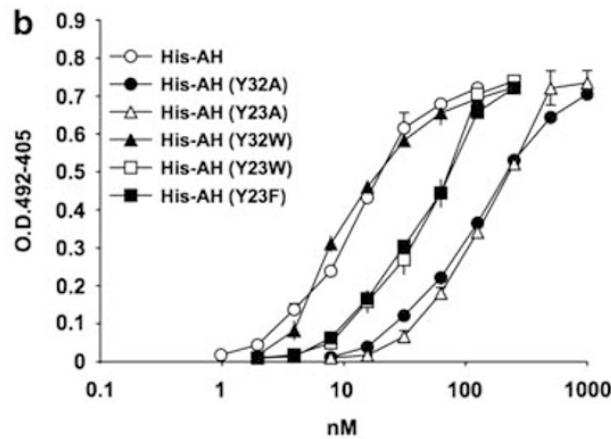

Figure 3 Enzyme-linked immunosorbent assay study of gp120-binding activities of Ala-substituted mutants (a) and Phe- or Trp-substituted mutants at positions 23 and 32 (b).
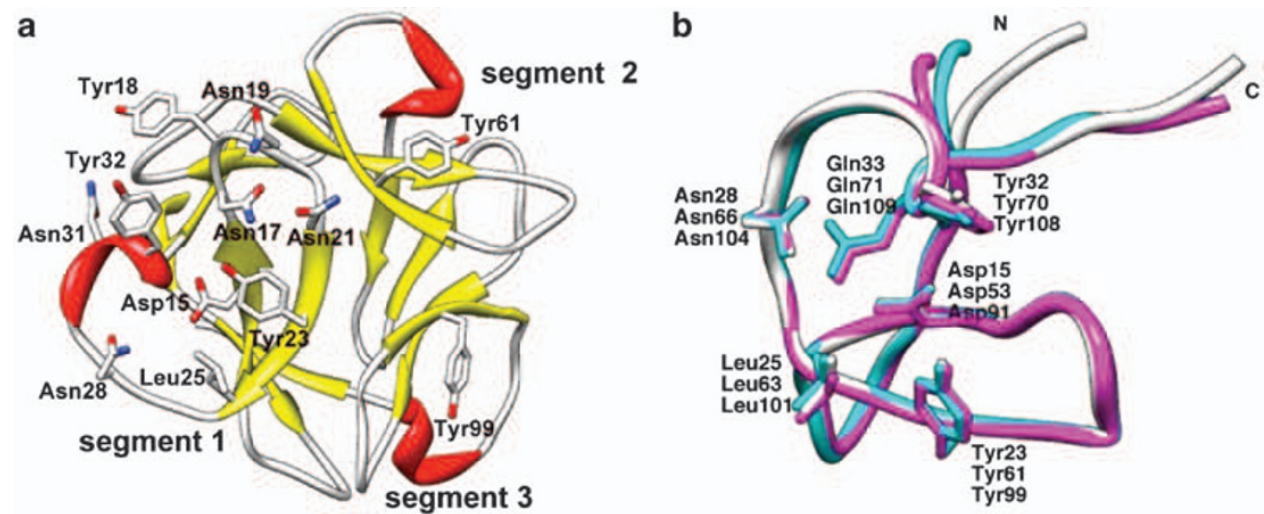

Figure $4 \mathrm{~A}$ whole view (a) of the tertiary structure of actinohivin (AH), and a superposition (b) of the three segments (modules), 1 (white), 2 (magenta) and 3 (cyan), of $\mathrm{AH}$. In (a), yellow arrows and red ribbons show $\beta$-strands and $\pi$-helices, respectively. The residues targeted for the present mutational analysis are labeled. In (b), the six active residues of segment 1 and corresponding residues in segments 2 and 3 are labeled. 
width and $3 \AA$ in depth) formed by a LD-QXW sequence in module 1 is a probable location for a sugar-binding pocket to accommodate one or two Man- $\alpha$ (1-2)-Man units of a HMTG. The role of valleys found in modules 2 and 3 is considered to be the same as that in the module $1 .^{12}$ These suggest that AH is able to accept three HMTGs on gp120. The three modules of $\mathrm{AH}$ assemble in a similar manner to the $\beta$-trefoil fold $^{13}$ that proteins belonging to CBM13 commonly possess, and this fold is also found in RTB and XBD. Although primary sequence homology between AH, RTB and XBD is low, overall three-dimensional structures are generally similar to each other. The LD-QXW sequences of $\mathrm{AH}$ have much higher intramolecular homology $(77 \%$ identity) than that of RTB or XBD (3 or 40\% identity, respectively). Furthermore, a superposition of the three-dimensional structures between the three modules of $\mathrm{AH}$ also shows that the overall structures of modules 2 and 3, including six residues corresponding to the active residues of module 1 , are strictly comparable to those of module 1 (Figure $4 \mathrm{~b}$ ). This identical nature indicates that three segments have equivalent specific HMTG-binding ability. Consequently, multivalent interactions between three segments and three HMTGs create an enhanced affinity of AH to gp120. This seems to be the crucial factor responsible for AH's potent anti-HIV property. The affinity enhancement through multivalent interactions between carbohydrate ligands and receptors is established via the glycoside cluster effect of lectin; ${ }^{23}$ the finding that mutant AHs lacking one or two segments showed reduced anti-syncytium formation activity reflects the cluster effect of lectin. ${ }^{20}$

In this study, amino acid residues in module 1 were chosen based on the highly conserved residues between the three segments, with mutants also exhibiting the six residues essential for gp120-binding activity. As shown in Figure 4b, the six residues of module 2, Asp53, Tyr 61, Leu63, Asn66, Tyr70 and Gln71, and residues, Asp91, Asp91, Tyr99, Leu101, Asn104, Tyr108 and Gln109 of module 3, are well fitted to the corresponding respective residues in module 1 , in both their positions and orientations. This means that activity data are comparable between modules. Indeed, in addition to Gln71 and Gln109, previously described, ${ }^{20}$ Tyr61 and Tyr99 were identified to be essential for AH (Table 2).

His-AH (Y23F), His-AH (Y23W) and His-AH (Y32W) show similar and slightly lower anti-syncytium formation activities compared with His-AH (Table 2). These data imply that aromatic rings at positions 23 and 32 are required for potent gp 120-binding affinity and those may participate in Man- $\alpha(1-2)$-Man binding through a stacking interaction with mannose rings. Our substitution analysis was actually undertaken for another purpose. In the case of the synthetic peptide derived from cellulose-binding domain of cellobiohydrolase I in the filamentous fungus Trichoderma reesei, it was reported that substitution of Trp for Tyr increased sugar-binding affinity, probably via stacking interaction formed with its substrate. ${ }^{24}$ This enhancement may be ascribed to higher hydrophobicity of the indole side chain of Trp than that of the phenol ring of Tyr, being a key factor in the aromatic ring-sugar interaction. ${ }^{25,26}$ Therefore, the substitutions of Trp for Tyr23 and Tyr32 of AH were expected to improve antisyncytium formation activity by enhancement of Man- $\alpha(1-2)$-Manbinding affinity. However, our assays found practically no increase. Tyr61 and Tyr70 of segment 2, and Tyr99 and Tyr108 of segment 3, corresponding to Tyr23 and Tyr32 of segment 1 , are all available to interact with mannose rings.

In conclusion, our research has identified the six amino acid residues of $\mathrm{AH}$ essential for anti-HIV activity. This will provide valuable insight into the AH-HMTG/gp120 binding mechanisms, as well as allowing rational design of more active $\mathrm{AH}$ derivatives, which should then expedite development of more effective and much-needed microbicides to help prevent HIV transmission.

\section{ACKNOWLEDGEMENTS}

This work was supported by the Japan Ministry of Education, Culture, Sports, Science and Technology grants from the 21st Century Program (to HT and SO), and the Japan Ministry of Health, Labor and Welfare Research fund from Japan Health Science Foundation on Drug Innovation (to HT). The authors thank Miss M Kamimura for her technical assistance.

1 AIDS epidemic update (2009) Joint United Nations Programme on HIV/AIDS and World Health Organization, (http://www.unaids.org/en/).

2 McGowan, I. Microbicides: a new frontier in HIV prevention. Biologicals 34, 241-255 (2006).

3 Leonard, C. K. et al. Assignment of intrachain disulfide bonds and characterization of potential glycosylation sites of the type 1 recombinant human immunodeficiency virus envelope glycoprotein (gp120) expressed in Chinese hamster ovary cells. J. Biol. Chem. 265, 10373-10382 (1990).

4 Rudd, P. M. \& Dwek, R. A. Glycosylation: heterogeneity and the 3D structure of proteins. Crit. Rev. Biochem. Mol. Biol. 32, 1-100 (1997).

5 Burton, D. R. A vaccine for HIV type 1: the antibody perspective. Proc. Natl Acad. Sci. USA 94, 10018-10023 (1997).

6 Botos, I. \& Wlodawer, A. Proteins that bind high-mannose sugars of the HIV envelope. Prog. Biophys. Mol. Biol. 88, 233-282 (2005).

7 Balzarini, J. Inhibition of HIV entry by carbohydrate-binding proteins. Antiviral Res. 71, 237-247 (2006)

8 Matsumoto, A. et al. Longispora albida gen. nov., sp. nov., a novel genus of the family Micromonosporaceae. Int. J. Syst. Evol. Microbiol. 53, 1553-1559 (2003).

9 Chiba, H. et al. A simple screening system for anti-HIV drugs: syncytium formation assay using T-cell line tropic and macrophage tropic HIV env expressing cell lines; establishment and validation. J. Antibiot. 54, 818-826 (2001).

10 Chiba, H. et al. Actinohivin, a novel anti-HIV protein from an actinomycete that inhibits syncytium formation: isolation, characterization and biological activities. Biochem. Biophys. Res. Commun. 282, 595-601 (2001).

11 Chiba, H ., Inokoshi, J., Nakashima, H., Omura, S .\& Tanaka, H. Actinohivin, a novel anti-human immunodeficiency virus protein from an actinomycete, inhibits viral entry to cells by binding high-mannose type sugar chains of gp120. Biochem. Biophys. Res. Commun. 316, 203-210 (2004).

12 Tanaka, H. et al. Mechanism by which the lectin actinohivin blocks HIV infection of target cells. Proc. Natl Acad. Sci. USA 106, 15633-15638 (2009).

13 Murzin, A. G., Lesk, A. M. \& Chothia, C. $\beta$-Trefoil fold patterns of structure and sequence in Kunitz inhibitor interleukins- $1 \beta$ and $1 \alpha$ and fibloblast growth factors. J. Mol. Biol. 223, 531-543 (1992).

14 Rutenber, E. \& Robertus, J. D. Structure of ricin B-chain at $2.5 \AA$ A resolution. Proteins. 10, 260-269 (1991).

15 Fujimoto, Z. et al. Crystal structure of Streptomyces olivaceoviridis E-86 $\beta$-xylanase containing xylan-binding domain. J. Mol. Biol. 300, 575-585 (2000).

16 Fujimoto, Z. et al. Crystal structure of sugar complexes of Streptomyces olivaceoviridis E-86 xylanase: sugar-binding structure of family 13 carbohydrate binding module. J. Mol. Biol. 316, 65-78 (2002).

17 Inokoshi, J., Chiba, H., Takahashi, A., Omura, S. \& Tanaka, H. Molecular cloning of actinohivin, a novel anti-HIV protein from an actinomycete, and its expression in Escherichia coli. Biochem. Biophys. Res. Commun. 281, 1261-1265 (2001).

18 Ho, S. N., Hunt, H. D., Horton, R. M., Pullen, J. K. \& Pease, L. R. Site-directed mutagenesis by overlap extension using the polymerase chain reaction. Gene 77, 51-59 (1989).

19 Laemmli, U. K. Cleavage of structural proteins during the assembly of the head of bacteriophage T4. Nature 227, 680-685 (1970).

20 Takahashi, A., Inokoshi, J., Chiba, H., Omura, S. \& Tanaka, H. Essential regions for antiviral activities of actinohivin, a sugar-binding anti-human immunodeficiency virus protein from an actinomycete. Arch. Biochem. Biophys. 437, 233-240 (2005).

21 Vyas, N. K. Atomic features of protein-carbohydrate interactions. Curr. Opin. Struct. Biol. 1, 732-740 (1991).

22 Quiocho, F. A. Probing the atomic interactions between proteins and carbohydrates. Biochem. Soc. Trans. 21, 442-448 (1993).

23 Lee, Y. C. \& Lee, R. T. Carbohydrate-protein interactions: basis of glycobiology. Acc. Chem. Res. 28, 321-327 (1995).

24 Linder, M., Lindeberg, G., Reinikainen, T., Teeri, T. T. \& Pettersson, G. The difference in affinity between two fungal cellulose-binding domains is dominated by a single amino acid substitution. FEBS Lett. 372, 96-98 (1995)

25 Reinikainen, T., Teleman, O. \& Teeri, T. T. Effects of $\mathrm{pH}$ and high ionic strength on the adsorption and activity of native and mutated cellobiohydrolase I from Trichoderma reesei. Proteins. 22, 392-403 (1995).

26 Engle, A. R., Purdie, N. \& Hyatt, J. A. Induced circular dichroism study of the aqueous solution complexation of cello-oligosaccharides and related polysaccharides with aromatic dyes. Carbohydr Res. 265, 181-195 (1994). 